\title{
Massive Star Formation Throughout the Galactic Disk
}

\author{
Stan Kurtz \\ Center for Radioastronomy and Astrophysics, National Autonomous University of Mexico, \\ Morelia, Michoacan 58089, Mexico \\ email: s.kurtz@crya.unam.mx
}

\begin{abstract}
High-mass star formation is manifestly a phenomenon of the Galactic Plane. The process begins with pre-stellar cores, evolves to proto-stellar objects, and culminates in massive main-sequence stars. Because massive young stellar objects are deeply embedded, the radio, sub$\mathrm{mm}$, and far/mid-infrared spectral windows are the most revealing. Galactic plane surveys at these wavelengths trace hot and cold molecular gas, interstellar masers, warm dust, and ionized gas that are present during star formation.
\end{abstract}

Keywords. surveys, stars: early type, stars: formation

Massive star formation is manifestly a phenomenon of the Galactic plane. All tracers of massive star formation are confined to the Galactic plane and are closely associated with the spiral arms.

The formation process of a high-mass star comprises four stages: 1) optically thin freefall collapse within a molecular cloud; 2) a hydrostatic core accreting onto a protostellar object; 3) optically thick hot molecular cores; and 4) entry onto the main-sequence, accompanied by the formation of an ultracompact HII region. These four stages are characterised by: 1) cold, dusty molecular clouds; 2) warm, dusty molecular clumps; 3) hot, dusty molecular cores; and 4) compact, ionized gas.

Various Galactic Plane Surveys are well-suited to trace these distinct evolutionary states. Infrared dark clouds were discovered in MSX images and SPITZER, AKARI and HERSCHEL are well-suited to detect warm dust. The ATLASGAL and JCMT Legacy surveys cover substantial portions of the Galactic plane in the sub-millimetre regime, that probes cool dust emission. The Multibeam Methanol Survey traces maser emission sites, while CORNISH will be sensitive to all ultracompact and many hypercompact HII regions within the survey area.

Galactic Plane Surveys contribute to high-mass star formation studies in several ways. The most obvious is to address questions of a statistical nature. The more accurate estimates of the numbers of hot molecular cores, ultracompact HII regions, and other objects will allow improved lifetime estimates. Surveys can also contribute to more detailed studies. For example, hypercompact HII regions will not be resolved by any of the infrared, sub-mm, or centimetre-wave surveys, but just having the flux densities over this large a range will be useful to constrain models of their density distributions. However, the interpretation of survey data will not always be clear. In fact, we will need to learn some new astrophysics to properly interpret some survey data. An example is the Methanol Multibeam Survey which will locate star formation sites via methanol maser emission. Although these masers are thought to trace early stages of massive star formation, it is not well-known precisely what stage they trace, or for how long they are present. These and other questions must be addressed before we will be able to fully understand what the surveys are telling us. 